\title{
Smart Technology for Mapping Obesity; Tracking Obesity for overuse of Smart Tehnology as a Risk factor Carolyn D. Rodgers*
}

Governors State University, Senior Lecturer Department of Addiction Studies and Behavioral Health College of Health and Human Services (CHHS) G, Building, Rm 136 University Park, IL, USA

\section{Introduction}

Obesity is an ongoing health concern that continues to be monitored as a precursor for negative health outcomes. Although the use of smart techonology has been accepted as an essential tool for improving health, there has been an increase in smart devices developed for obesity prevention and weight reduction. These smart devices such as smart phones, computers, tablets, heart monitors, chest pins, and more are used for lifestyle modifications that attempt to improve health outcomes without the interferrence of human interaction.

\section{Smart Technology and Human Abilities}

The use of smart devices are often created for convienience to elimate the amount of man-hours required to collect, record, and analyze data for evaluation purposes. However, human error are factors than can hinder progress and positive health outcomes. Simultaneously, these same devices are often created to be objective, obscure, and effective in monitoring human behavior $[1,2]$. Similarly, smart devices also have the ability to record more elaborate inputs, where human observation is not as invasive. Contrariwise, using smart devices can inflict a privacy issue in data collection for human subjects. More importantly, smart devices are rigid and does not recognize external factors that a human can. Likewise, smart technology has a limit that a can impede or stop data collection altogether if not operated properly. Nevertheless, smart technology is having a huge impact on the way healthcare is implemented, measured and reported, especially for preventable diseases and disorders such as obesity.

\section{Obesity Prevention and Monitoring}

In terms of obesity prevention and weight reduction, there are several factors that must be present in order to reach a positive outcome. Firstly, motivation to make a change which is the thriving force to begin any process [3], and the will power to make a commitment [4]. Secondly, setting realistic goals to make the necessary changes for obesity prevention or weightloss and lifestyle monitoring $[1,2]$ are essential for obtaining sucessful results. Alternatively, several studies have shown success using smart technology for obesity prevention hightlighting factors previous mentined. For example, Glynn, et al, [2] used smart phones with accelerator applications to provide outcome data regarding step count measurements as primary care to increase physical activity for individuals over 16 years of age with smartphones. Another research team, Smith, Morgan, Plotnikoff, Dally, Salmon, Okely [4] also used smart phones for obesity prevention in adolesescent boys and to include and encourgage physical activity. Furthermore, an exploratory study conducted by Sun, Burke, Baranowski, Fernstrom, Zhang [1] used an electronic wearable chest device to monitor and evaluate diet and lifestyle. The chest pin was developed as a means to be objective, convienient, and to moniotor human dietary lifestyle choices [1]. Although smart technology is being used to reduce obesity and increase weightloss, there are concerns for overuse of smart technolgy and risk for obesity.

\section{Smart Technology as a Vice for Obesity}

Overuses of smart devices are associated with increased risk for obesity and are influencers of making unhealthy decisions. Researchers, Bornhorst, Wijnhoven, Kunesova, Yngve, Rito, Lissner [5] conducted a study based on parent-reported screen time, sleeping patterns and diet consumption of children aged 6 to 9 to determine that screen time was associated with increased sugar consumption. Likewise, increased screen time also has been shown to negatively affect sleep duration $[5,6]$ a direct link to risk for obesity. Still, having ties to social networks and increased social interactions has been shown to decrease risk for obesity and weight gain, as people are often influenced by their social group [6,7]. Albeit, smart technology has its place in public health as it has proven to be useful and beneficial in many settings.

\section{Assumption in Moderation}

In summary, Smart technology has it places for obesity prevention, behavior modification, and lifestyle changes. However, a healthy balance for using smart technology needs to be recognized. As smart technology is convenient and should be used for supplementation to human efforts and not as a total replacement for human responsibilities. Although, it is recognized that human and machines alike have flaws, such as recall bias, and systematic error; it is only when both are recognized for their strengths, weaknesses, and collaborative uses that healthy outcomes are achieved.

\section{References}

1. Sun M, Burke LE, Baranowski T, Fernstrom JD, Zhang H, et al. (2015) An exploratory study on a chest-worn computer for evaluation of diet, physical activity and lifestyle. J Healthc Eng 6: 1-22.

2. Glynn LG, Hayes PS, Casey M, Glynn F, Alvarez-lglesias A, et al. (2013) SMART MOVE - a smartphone-based intervention to promote physical activity in primary care: study protocol for a randomized controlled trial. Trials14: 157.

3. Styn MA, Wang J, Acharya SD, Yang K, Chasens ER, et al. (2013) Healthrelated quality of life among participants in the SMART weight loss trial. App Nurs Res 25: 276-279.

4. Smith JJ, Morgan PJ, Plotnikoff RC, Dally KA, Salmon J, et al. (2015) Smartphone obesity prevention trial for adolescent boys in low-income communities: the ATLAS RCT. Pediatrics 134: e723-731.

5. Bornhorst C, Wijnhoven T, Kunesova MA, Yngve M, Rito Al, et al. (2015) WHO childhood obesity surveillance initiative: associations between sleep duration, screen time and food consumptions frequencies. BMC Public Health 15: 422.

6. Borghese MM, Tremblay MS, Katzmarzyk PT, Tudor-Locke C, Schuna JM Jr et al. (2015) Mediating role of television time, diet patterns, physical activity and sleep duration in the association between television in the bedroom and adiposity in 10 year-old children. Int J Behav Nutr Phys Act 12: 60.

7. Oloritun RO, Ouarda TB, Moturu S, Madan A, Pentland AS, et al. (2013) Change in BMl accurately predicted by social exposure to acquaintances. PLoS One : e79238.

*Corresponding author: Carolyn D. Rodgers, Governors State University, Senior Lecturer, Department of Addiction Studies and Behavioral Health College of Health and Human Services (CHHS) G, Building, Rm 136 University Park, IL, USA, Tel: 708-235-7394; E-mail: cestes@govst.edu

Received September 19, 2015; Accepted September 21, 2015; Published September 25, 2015

Citation: Rodgers CD (2015) Smart Technology for Mapping Obesity; Tracking Obesity for overuse of Smart Tehnology as a Risk factor. J Health Med Informat 6: e138. doi:10.4172/2157-7420.1000e138

Copyright: (c) 2015 Rodgers CD. This is an open-access article distributed under the terms of the Creative Commons Attribution License, which permits unrestricted use, distribution, and reproduction in any medium, provided the original author and source are credited. 